\title{
Individual differences in the perceptual span during reading: Evidence from the moving window technique
}

\author{
Wonil Choi $^{1}$ - Matthew W. Lowder ${ }^{1}$ - Fernanda Ferreira ${ }^{1}$. \\ John M. Henderson ${ }^{1}$
}

Published online: 10 June 2015

(C) The Psychonomic Society, Inc. 2015

\begin{abstract}
We report the results of an eye tracking experiment that used the gaze-contingent moving window technique to examine individual differences in the size of readers' perceptual span. Participants read paragraphs while the size of the rightward window of visible text was systematically manipulated across trials. In addition, participants completed a large battery of individual-difference measures representing two cognitive constructs: language ability and oculomotor processing speed. Results showed that higher scores on language ability measures and faster oculomotor processing speed were associated with faster reading times and shorter fixation durations. More interestingly, the size of readers' perceptual span was modulated by individual differences in language ability but not by individual differences in oculomotor processing speed, suggesting that readers with greater language proficiency are more likely to have efficient mechanisms to extract linguistic information beyond the fixated word.
\end{abstract}

Keywords Perceptual span · Individual differences · Eye movements $\cdot$ Reading

\section{Introduction}

Individual differences on a variety of cognitive tasks have been shown to relate to variability in eye movements during reading (e.g., Ashby, Rayner, \& Clifton, 2005; Jared, Levy, \& Rayner, 1999; Kuperman \& Van Dyke, 2011). A key

Wonil Choi

wichoi@ucdavis.edu

1 Center for Mind and Brain, University of California, Davis, CA 95618, USA requirement for explaining the nature of individual differences during reading is to better understand how and why readers differ in their ability to extract visual information from the parafovea and to use this information to make processing more efficient. The role of parafoveal processing during reading is often examined using the gaze-contingent moving window paradigm (McConkie \& Rayner, 1975; Rayner, 1975), in which participants are presented with text that is masked except within a predefined window extending to the left and right of fixation. The window follows fixation as the reader moves through the text. Reading rate is impaired at very small window sizes, but tends to increase as the size of the window increases. Using this technique, researchers can establish the perceptual span for a particular reader - that is, the window size at which reading rate is the same as reading rate without a window. The perceptual span for skilled readers of English tends to be around 3-4 characters to the left and 14-15 characters to the right of fixation (for a review, see Rayner, 2014).

The size of a reader's perceptual span has been found to depend on a variety of factors, including properties of the text (Apel, Henderson, \& Ferreira, 2012; Henderson \& Ferreira, 1990; Rayner, 1986), as well as properties of the individual (Ashby, Yang, Evans, \& Rayner, 2012; Häikiö, Bertram, Hyönä, \& Niemi, 2009; Rayner, 1986; Rayner, Slattery, \& Bélanger, 2010; Veldre \& Andrews, 2014). For example, Veldre \& Andrews (2014) showed that the size of the perceptual span was modulated by reading and spelling ability such that readers with lower scores tended to have shorter perceptual spans than readers with higher scores. Veldre and Andrews (2014) argued that readers who have more sophisticated orthographic knowledge are able to more rapidly access the lexical entries of the words they fixate, which enables them to use visual information in the parafovea more effectively. Kuperman and Van Dyke (2011) also proposed that highquality language skills are critical for efficient processing 
during reading, although they reported that measures assessing lexical decoding and rapid naming were the best predictors of a wide range of eye movement measures.

In the present study, we tested readers on a battery of individual difference measures representing two broad cognitive constructs: language ability and oculomotor processing speed. We used a variety of measures to compute each of these constructs under the assumption that this approach allows us to obtain a more reliable estimate of these skills than if we had used just a single measure. The measures we used to assess language ability included a range of tests that we hypothesized would reflect several different components of comprehension, including word decoding, listening comprehension ability, and print exposure. Decoding refers to the ability to translate a word's orthographic form into a phonological code, which is believed to be one of the most crucial factors involved in learning to read (McCardle \& Pugh, 2009; Rieben \& Perfetti, 1991). Kuperman and Van Dyke (2011) found that the score on a measure of word decoding (the Word Identification test from the Woodcock-Johnson-III Tests of Achievement) was one of the strongest predictors of eye movement behavior during reading. The second aspect of language ability that we examined was language comprehension ability, using the Listening Comprehension subtest of the Woodcock Reading Mastery Test. Although standardized measures of reading and listening comprehension are strongly correlated (Palmer, MacLeod, Hunt, \& Davidson, 1985), we chose to assess readers' comprehension skill using a Listening Comprehension test because the other measures we used to estimate language ability were more specific to reading, and we wanted a measure of comprehension that would not be redundant with those measures. Finally, we considered the role of print exposure. Many prior studies have used the Author Recognition Test (ART; Stanovich \& West, 1989) as a measure of an individual's exposure to print, showing that ART scores are correlated highly with vocabulary knowledge, declarative knowledge, reading comprehension, academic performance, and logical reasoning (Cunningham \& Stanovich, 1990, 1991, 1997; Stanovich \& West, 1989; Acheson, Wells, \& MacDonald, 2008; West, Stanovich, \& Mitchell, 1993; for a comprehensive review, see Mol \& Bus, 2011). Acheson et al. (2008) reported that, although measures of print exposure were correlated significantly with reading-related performance measured by verbal ACT scores (Stanovich, West, \& Harrison, 1995), the correlations between print exposure and online measures of reading ability such as reading rate (as measured by a self-paced reading task) and comprehension accuracy were not reliable. In the current study, we re-examined the relationship between print exposure and online measures of reading by employing eye tracking methodology, which allows us to examine natural reading processes.
The second cognitive construct that we hypothesized might affect the perceptual span is oculomotor processing speed. Several previous studies have shown that measures of oculomotor efficiency are related to measures of reading ability (e.g., Eden, Stein, Wood, \& Wood, 1994; Griffin, Walton, \& Ives, 1974; Juola, Haugh, Trast, Ferraro, \& Liebhaber, 1987; Lefton, Nagle, Johnson, \& Fisher, 1979; Pavlidis, 1981, 1983; for a review, see Kulp \& Schmidt, 1996). For example, Griffin et al. (1974) demonstrated that individuals with poor reading skills also showed poor oculomotor control in a non-reading eye movement task relative to more skilled readers, suggesting that there might be a tight link between oculomotor processing ability and reading ability. However, others have rejected the notion that non-reading oculomotor differences are an effective predictor of reading ability, noting that these findings have been difficult to replicate (see, e.g., Eskenazi \& Diamond, 1983; Olson, Kliegl, \& Davidson, 1983; Rayner, 1985; Stanly, Smith, \& Howell, 1983). Given this inconsistency, it may be informative to examine whether individual differences in oculomotor processing speed are related to variability in the perceptual span during reading.

Recently, Rayner et al. (2010) reported that faster readers had larger perceptual spans relative to slower readers, suggesting that reading rate is a critical factor to consider. Because it is possible that fast reading is associated with faster oculomotor processing in general, the current study assessed oculomotor processing speed with two different tasks: rapid automatized naming (RAN) and a circle targeting task. RAN tasks require participants to rapidly name a series of items presented in an array, and these tasks have been used extensively in studies of children's reading development (Denckla \& Rudel, 1974, 1976; see Swanson, Trainin, Necoechea, \& Hammill, 2003, for a meta-analysis). Some studies have indicated that RAN performance reflects processing speed above and beyond reading ability (Cutting \& Denckla, 2001; Powell, Stainthorp, Stuart, Garwood, \& Quinlan, 2007; Kail \& Hall, 1994; Kail, Hall, \& Caskey, 1999; Savage, Pillay, \& Melidona, 2007; Wolf \& Bowers, 1999). Kuperman and Van Dyke (2011) demonstrated that RAN performance was one of the strongest unique predictors of eye-movement behavior during reading and suggested that the RAN task should be considered an index of oculomotor processing speed, which allows rapid coordination of lexical processing and eyemovement control (e.g., Reichle, Pollatsek, Fisher, \& Rayner, 1998). In addition to the RAN task, we used a circle targeting task in which participants were asked to fixate a row of circles presented on a computer screen serially (moving from left to right or right to left) as rapidly as possible. We hypothesized that this task would reflect readers' speed of processing and oculomotor control without any involvement of language processing. If oculomotor processing speed is a critical factor modulating eye movement control during 
reading, then we would expect to obtain evidence that faster oculomotor processing is associated with a larger perceptual span.

In summary, in the present study, we assessed the influence of individual difference measures indexing language ability and oculomotor processing speed on eye movements during reading, focusing in particular on variability in the size of readers' perceptual spans. Our hypothesis was that stronger language abilities and faster oculomotor processing are associated with larger perceptual spans in reading.

\section{Method}

\section{Participants}

Seventy undergraduate students from the University of South Carolina participated in the experiment in exchange either for course credit or for US \$8. All had normal or corrected-to-normal vision and were native speakers of English.

\section{Materials and design}

The experimental materials consisted of 80 short paragraphs (ranging in length from 42 to 61 words) selected from the USA Today online archive (available at http://pqasb.pqarchiver. com/USAToday/search.html). These paragraphs contained 2,328 unique words with a total of 4,140 word tokens.

We manipulated the rightward window size and kept the leftward window size constant across conditions at four characters. The size of the rightward window was systematically manipulated at lengths of 4 characters, 8 characters, 12 characters, and 16 characters. We also included a no-window control condition in which there were no viewing restrictions on either the left or right of fixation. These five viewing conditions were manipulated within subjects, such that each participant read 16 of the paragraphs under each of the five conditions, presented in a random order. The paragraphs were fully counterbalanced over all conditions across participants.

\section{Measures of individual differences in language ability}

All participants completed a battery of individual difference measures.

\section{Author recognition test}

In the author recognition test (ART), participants were presented with a list of names and asked to mark the ones they recognize as authors (Stanovich \& West,
1989). The test contained 65 author names and 65 foils, and scores were calculated by subtracting the number of foils marked from the number of real authors marked. We used the most recent version of the ART (Acheson et al., 2008).

\section{Comparative reading habits}

The comparative reading habits $(\mathrm{CRH})$ questionnaire consisted of five questions that asked about an individual's reading habits compared to those of other college students (see Acheson et al., 2008, for a detailed description). Each question was measured on a sevenpoint Likert scale. The questions assessed individuals' perceptions of their time spent reading, the complexity of their reading material, reading enjoyment, reading rate, and reading comprehension ability.

\section{Word and nonword decoding (basic skills)}

Subsets of the Woodcock Reading Mastery Tests-III (WRMT-III) were used to assess decoding ability of words and nonwords (Woodcock, 2011). The split-half reliability of the two tests is high across age (.92 for Word Identification and .88 for Word Attack), and these tasks correlate highly with other measures of reading ability (e.g., $r=.80$ between scores of the Basic Skills in the WRMT-III and those in the WRMT-Revised Normative Update (R/NU), Woodcock, 2011).

\section{Word identification}

In this test, participants were asked to read isolated words aloud. Participants were instructed to read the words whether or not they knew their meanings. The test consists of 46 trials; however, participants in the current study started at trial number 30, which is the starting point for high school students and adults. All administrated trials were given a score of 1 or 0 . Each individual's score was calculated by adding the number of trials answered correctly to the number of unexamined trials below the starting point.

\section{Word attack}

The procedure for Word Attack was identical to that of Word Identification except that the items were nonwords. There are 26 trials in total, but participants started this test from the tenth trial, which is the starting point for high school students and adults. The same scoring method to that of the Word Identification test was applied to this test. 


\section{Listening comprehension}

The listening comprehension subtest of the WRMT-III was used to assess participants' comprehension ability. The split-half reliability $(r=.93)$ and validity measured by correlations with similar types of tests from other measures of reading skill are relatively high $(\mathrm{r}=.84$ between WRMT-III and WRMT-R/NU, Woodcock, 2011). Participants listened to short passages and were asked a comprehension question after each passage. There were 27 trials in total, but participants started this test from the 13th trial, which is the starting point for high school students and adults.

\section{Rapid automatized naming}

In the RAN task, participants are presented with a $6 \times 6$ grid of items and asked to name each item in the array as quickly as possible. We used the four RAN subtests of the WRMT III, which assesses naming of letters, digits, colors, and pictures of objects. Response times were measured from when the participant began naming the first item of an array until the last item in the array was named. The split-half reliability was high $(r=.83$ for the combined object naming and color naming tests, and $\mathrm{r}=.88$ for the combined digit naming and letter naming tests). In addition, validity measured by correlations with similar types of tests from other measures of reading skill are high $(\mathrm{r}=.78$ between WRMT-III and CTOPP, Woodcock, 2011).

\section{Circle targeting task}

This task included two display types, one with 20 circles displayed in a $2 \times 10$ array, and the other with 40 circles displayed in a $4 \times 10$ array. There were ten trials in each display type. The size of the circles was equivalent to the size of one character in the reading task (see below). Participants were asked to fixate every circle on the screen as rapidly as possible, both in the leftto-right and right-to-left direction. The dependent measure was the total time required to fixate each circle in the trial, and was recorded by the eye tracker.

\section{Apparatus}

An Eyelink 1000 eye tracker (SR Research, Osgoode, ON, Canada) was used to record participants' eye movements. Viewing was binocular, but only the right eye was monitored at a sampling rate of $1000 \mathrm{~Hz}$. Stimuli were presented on a ViewSonic G225f monitor with a screen resolution of $1024 \times 768$ pixels and with a $120 \mathrm{~Hz}$ monitor refresh rate. Participants were seated $85 \mathrm{~cm}$ away from the monitor, and a head rest and a chin rest were used to minimize head movement. Paragraphs were presented in black on a white background using $16 \mathrm{pt}$ Courier New font (a monospaced font). Circles in the circle targeting task were also presented in black on a white background using 16 pt Courier New font. Each letter extended 12.75 pixels horizontally, so that 2.98 characters subtended about $1^{\circ}$ of visual angle. The experiment was controlled using Experiment Builder software (SR Research).

\section{Procedure}

For the moving window experiment, participants were instructed to read paragraphs for comprehension. The eye tracker was calibrated at the start of each session using a nine-point grid, and the calibration procedure was re-run after every 20 experimental trials. At the beginning of each trial, participants fixated a dot marking the position where the initial character of the paragraph would appear. When fixation on this point was stable, the experimenter presented the paragraph. Participants first read five practice paragraphs, and then the 80 experimental paragraphs were presented in a different random order for each participant. A yes-or-no comprehension question was presented on all practice trials and a quarter of the experiment trials. Mean accuracy on the comprehension questions was $80.6 \%$ and did not vary as a function of window size.

After the moving window experiment, the eye tracker was recalibrated and participants performed the circle targeting task. At the beginning of each trial a fixation dot was presented in the position where the initial circle of the array would appear. When fixation on this point was stable, the experimenter presented the array of circles. Participants were first presented with two practice arrays, and then they were presented with the 20 experimental trials in a random order. Participants were instructed to fixate every circle in each array and to regress if they skipped any circle. After completing the eye tracking experiments, participants were presented with the remaining individual differences measures. The total time to complete the entire session was about $90 \mathrm{~min}$.

\section{Results}

\section{Analysis}

For the reading data, fixations shorter than $50 \mathrm{~ms}$ or longer than $1500 \mathrm{~ms}$ were excluded. In addition, fixations were excluded if they immediately preceded or 
Table 1 Means, standard deviations, and ranges of each individual difference test. Raw scores of Word identification, Word attack, and listening comprehension were converted to standardized scores based on the results of a large-scale norming study (Woodcock, 2011). RAN Rapid automized naming

\begin{tabular}{lll}
\hline Test & Mean (SD) & Range \\
\hline Author recognition test & $11.2(5.96)$ & $1-27$ \\
Comparative reading habits & $4.32(1.07)$ & $1.4-6$ \\
$\quad$ Word identification & $100.79(12.38)$ & $77-134$ \\
$\quad$ (standardized score) & $95.63(12.36)$ & $68-121$ \\
$\quad$ Word attack (standardized score) & $107.73(12.07)$ & $64-122$ \\
$\quad$ Listening comprehension & $19.62(3.25)$ & $13.66-30.28$ \\
$\quad$ (standardized score) & $18.23(2.73)$ & $13.06-24.97$ \\
Object RAN (s) & $11.88(2.45)$ & $6.94-19.44$ \\
Color RAN (s) & $11.68(2.12)$ & $7.75-18.22$ \\
Number RAN (s) & $16.48(4.08)$ & $4.16-27.36$ \\
Letter RAN (s) & & \\
Circle targeting task (s) & &
\end{tabular}

followed a blink, or if their saccade amplitude before the fixation was more than $15^{\circ}$. Finally, fixations that occurred during track losses were excluded. In total, $16.2 \%$ of the fixations were excluded from the analysis.

We examined reading rate measured in words per minute (WPM), which is the dependent variable most commonly used in the moving window paradigm (Rayner, 2014). In addition, mean fixation duration, mean forward saccadic amplitude (in degrees), and mean regression count per trial were also analyzed.

Descriptive statistics of all individual difference measures are presented in Table 1. Because several of these measures were moderately or highly correlated with one another (see Appendix 1), two composite variables were generated. First, all individual difference measures were submitted to a principal components analysis (PCA) with the varimax rotation method. Based on the component loadings of the PCA, we identified two principal factors (see Appendix 2 for the detailed results of the PCA). The first factor, which includes performance on the four RAN subtests and the circle targeting task, reflects oculomotor processing speed. The second factor, which includes scores on the remaining measures, reflects language ability. Then, composite scores representing these two factors were created for each participant by standardizing and averaging the individual scores (oculomotor processing speed: mean $=-.01, \mathrm{SD}=.78$, range $=-1.94$ to 1.58; language ability: mean $=.03, \mathrm{SD}=.73$, range from 1.43 to 1.64$)$. The two composite scores were moderately correlated $(r=-.286, P=.016)$. Our analyses focused primarily on these two composite variables; however, we also present basic correlational analyses of the relationships between the separate individual differences measures and eye tracking measures.

The data were analyzed using linear mixed effects (LME) models with the lmer function in the lme4 package (Bates, Maechler, \& Bolker, 2012) in R (R Development Core Team, 2011), with subjects and items entered as crossed random effects. The lmerTest package (Kuznetsova, Brockhoff, \& Christensen, 2013) was used to obtain all $P$ values. Fixed effects included window size, the composite factor for processing speed, the composite factor for language ability, and the interactions among these factors. For the fixed effect of window size, successive difference contrasts (Venables \& Ripley, 2002) were tested across each level of this factor to evaluate the effect of systematic increases in window size. Random effects included intercepts for subjects and items. By-subject and by-item random slopes were not included in the analyses because the models including them failed to converge.

The remainder of this section is organized in three parts. First, we describe basic relationships between reading time data and our individual differences measures using data obtained from the no-window condition. Then, we examine the effect of window size on eye movements. Finally, we report analyses designed to investigate how individual differences modulate the effect of window size on eye movements.

\section{Correlations between individual difference measures and eye movement measures}

Table 2 shows correlations between individual difference measures and mean fixation duration, forward saccadic amplitude, regression count, and WPM in the nowindow condition. Several of the measures that reflect language ability showed moderate-to-strong correlations with fixation duration, saccadic amplitude, and words per minute. In general, higher scores on these measures were associated with shorter fixation durations, larger saccadic amplitude, and more words read per minute. Regarding measures that reflect speed of processing, RAN was related to several eye movement measures, such that faster performance on the RAN was associated with shorter fixation durations, fewer regressive saccades, and faster reading in WPM. In addition, faster performance on the circle targeting task was associated with faster reading rate in WPM.

\section{Overall eye movement data}

Mean values for the dependent variables across window size conditions are reported in Table 3 , and inferential 
Table 2 Correlations between individual-difference measures and eye-movement measures in the no-window condition. WPM Words per minute (reading rate measure)

\begin{tabular}{lllll}
\hline & Fixation duration $(\mathrm{ms})$ & Saccadic amplitude $\left({ }^{\circ}\right)$ & Regression count & $.41^{* * *}$ \\
\hline Author recognition test & $-.33^{* *}$ & $.43^{* * *}$ & .09 & $.44^{* * *}$ \\
Comparative reading habits & $-.42^{* * *}$ & $.41^{* * *}$ & .06 & $.32^{* *}$ \\
Word identification & $-.30^{*}$ & $.26^{*}$ & -.02 & .02 \\
Word attack & $-.35^{* *}$ & .23 & .21 & $.22^{* *}$ \\
Listening comprehension & -.19 & $.28^{*}$ & .14 \\
Mean of four RAN subtests & $.34^{* *}$ & -.17 & .22 & $-.42^{* * *}$ \\
Circle targeting task & .20 & -.09 & -.13 & $.30^{*}$ \\
Composite score of language ability & $-.34^{* *}$ & -.21 & $-.35^{* *}$ \\
Composite score of processing speed & .11 & & $-.28^{*}$ \\
\hline
\end{tabular}

${ }^{*} P<.05 ;{ }^{* *} P<.01 ; * * * P<.001$

${ }^{\mathrm{a}}$ The outcome measure of the RAN and the circle targeting tests is total time to complete the task so that bigger numbers represent slower performance

statistics are presented in Table 4. As seen in Tables 3 and 4 , reading rate (WPM) increased as window size increased. Additionally, fixation durations decreased and saccadic amplitude increased as the window size became larger. The number of regressive eye movements per trial significantly decreased from the 4character window to the 8-character window.

Interestingly, there were significant differences in several eye movement measures between the 16character window and the no-window condition such that there were faster reading rates, shorter fixation durations, and longer saccadic movements in the nowindow condition relative to the 16-character window condition. This result is inconsistent with the notion that a reader's perceptual span is about 15 characters to the right and that no additional benefit is obtained beyond the 15-character window (Rayner, 2009, 2014). Interestingly, Veldre and Andrews (2014) also found that there were additional benefits for a nowindow condition compared to a 15-character window condition when no individual difference measures were considered, providing further suggestive evidence that the perceptual span may be larger than has generally been assumed. We discuss this finding in greater detail in the Discussion.

\section{Eye movement data modulated by individual difference measures}

Table 4 shows that there were significant overall effects of the composite language ability measure on WPM, fixation duration, and saccadic amplitude, and that there were main effects of the composite processing speed measure on WPM, fixation duration, and number of regressive fixations. These results indicate that individual differences in language ability and processing speed modulate eye movements during reading, consistent with the correlational analyses reported above. Next, we describe how these constructs influence eye movement measures across the different window size conditions.
Table 3 Means of the eye movement measures (standard deviations in parentheses) for each window condition averaged over subjects. $4 \mathrm{~L} 4$ character-leftward window, $4 R$ 4-character-rightward window, $8 R$ 8character-rightward window, $12 R$ 12-character-rightward window, $16 R$ 16-character-rightward window; FixDur mean fixation duration, SacAmp mean forward saccade amplitude, RegCount number of regressive fixations per trial

Window condition

\begin{tabular}{llllll} 
& 4L4R & 4L8R & 4L12R & 4L16R & No window \\
\hline WPM & $189(38)$ & $221(49)$ & $233(53)$ & $235(57)$ & $244(64)$ \\
FixDur (ms) & $238(24)$ & $224(23)$ & $221(24)$ & $220(24)$ & $211(26)$ \\
SacAmp $\left(^{\circ}\right)$ & $2.17(.33)$ & $2.42(.39)$ & $2.53(.40)$ & $2.60(.46)$ & $2.64(.49)$ \\
RegCount & $12(5.6)$ & $11.25(5.5)$ & $11.47(5.8)$ & $11.69(5.9)$ & $11.43(5.5)$ \\
\hline
\end{tabular}


Table 4 Linear mixed effects analysis of the experiment

\begin{tabular}{|c|c|c|c|c|c|c|c|c|c|c|c|c|}
\hline \multirow[b]{2}{*}{ Model parameters } & \multicolumn{3}{|c|}{ Reading rate } & \multicolumn{3}{|c|}{ Fixation durations } & \multicolumn{3}{|c|}{ Forward saccade amplitude } & \multicolumn{3}{|c|}{ Regression count } \\
\hline & $\mathrm{b}$ & $\mathrm{SE}$ & $\mathrm{t}$ & $\mathrm{b}$ & SE & $\mathrm{t}$ & $\mathrm{b}$ & SE & $\mathrm{t}$ & $\mathrm{b}$ & SE & $\mathrm{t}$ \\
\hline (Intercept) & 221.704 & 5.470 & $40.533^{*}$ & 222.7405 & 2.5561 & $87.139 *$ & $2.45 \mathrm{E}+00$ & $4.52 \mathrm{E}-02$ & $54.26^{*}$ & 11.65533 & 0.50722 & $22.979 *$ \\
\hline Language ability & 21.333 & 7.237 & $2.948^{*}$ & -12.0714 & 3.5378 & $-3.412 *$ & $1.78 \mathrm{E}-01$ & $6.22 \mathrm{E}-02$ & $2.861^{*}$ & -0.16285 & $6.85 \mathrm{E}-01$ & -0.238 \\
\hline Processing speed & -21.111 & 6.836 & $-3.088^{*}$ & 7.05173 & 3.3414 & $2.110^{*}$ & $-3.28 \mathrm{E}-02$ & $5.87 \mathrm{E}-02$ & -0.558 & 1.59250 & $6.47 \mathrm{E}-01$ & $2.464 *$ \\
\hline Window (4 vs. 8 ) & 31.686 & 1.858 & $17.049^{*}$ & -14.2673 & 0.6840 & $-20.857^{*}$ & $2.50 \mathrm{E}-01$ & $1.20 \mathrm{E}-02$ & $20.892^{*}$ & -0.70183 & 0.15706 & $-4.468 *$ \\
\hline Window (8 vs. 12 ) & 12.787 & 1.855 & $6.893^{*}$ & -3.33457 & 0.6828 & $-4.884^{*}$ & $1.09 \mathrm{E}-01$ & $1.19 \mathrm{E}-02$ & $9.089^{*}$ & 0.20953 & 0.15677 & 1.337 \\
\hline Window (12 vs. 16) & 1.200 & 1.854 & 0.647 & -0.33135 & 0.6823 & -0.486 & $6.57 \mathrm{E}-02$ & $1.19 \mathrm{E}-02$ & $5.505^{*}$ & 0.19123 & 0.15677 & 1.221 \\
\hline Window (16 vs. NW) & 9.134 & 1.854 & $4.927 *$ & -8.67099 & 0.6824 & $-12.708^{*}$ & $3.92 \mathrm{E}-02$ & $1.19 \mathrm{E}-02$ & $3.282 *$ & -0.29646 & 0.15678 & -1.892 \\
\hline $\begin{array}{l}\text { Window (4 vs. } 8) \times \\
\text { Language ability }\end{array}$ & 6.940 & 2.670 & $2.599 *$ & -0.87056 & 0.9818 & -0.887 & $6.49 \mathrm{E}-02$ & $1.72 \mathrm{E}-02$ & $3.778^{*}$ & 0.34011 & 0.22562 & 1.507 \\
\hline $\begin{array}{c}\text { Window }(8 \text { vs. } 12) \times \\
\text { Language ability }\end{array}$ & -1.124 & 2.666 & -0.422 & -0.03245 & 0.9803 & -0.033 & $6.96 \mathrm{E}-03$ & $1.72 \mathrm{E}-02$ & 0.406 & -0.36711 & 0.22528 & -1.630 \\
\hline $\begin{array}{c}\text { Window (12 vs. } 16) \times \\
\text { Language ability }\end{array}$ & 6.167 & 2.667 & $2.312^{*}$ & -3.60133 & 0.9807 & $-3.672 *$ & $7.90 \mathrm{E}-02$ & $1.72 \mathrm{E}-02$ & $4.605^{*}$ & 0.45617 & 0.22537 & $2.024 *$ \\
\hline $\begin{array}{l}\text { Window (16 vs. NW) } \times \\
\text { Language ability }\end{array}$ & 7.340 & 2.669 & $2.750^{*}$ & -2.42115 & 0.9813 & $-2.467^{*}$ & $3.52 \mathrm{E}-02$ & $1.72 \mathrm{E}-02$ & $2.050^{*}$ & -0.11545 & 0.22553 & -0.512 \\
\hline $\begin{array}{l}\text { Window }(4 \text { vs. } 8) \times \\
\text { Processing speed }\end{array}$ & -4.127 & 2.529 & -1.632 & 0.96860 & 0.9298 & 1.042 & $2.06 \mathrm{E}-02$ & $1.63 \mathrm{E}-02$ & 1.267 & 0.19855 & 0.21372 & 0.929 \\
\hline $\begin{array}{c}\text { Window }(8 \text { vs. } 12) \times \\
\text { Processing speed }\end{array}$ & -1.540 & 2.514 & -0.612 & 0.89875 & 0.9243 & 0.972 & $-2.93 \mathrm{E}-03$ & $1.62 \mathrm{E}-02$ & -0.181 & -0.06781 & 0.21245 & -0.319 \\
\hline $\begin{array}{c}\text { Window }(12 \text { vs. } 16) \times \\
\text { Processing speed }\end{array}$ & -2.719 & 2.517 & -1.080 & -0.51424 & 0.9253 & -0.556 & $2.58 \mathrm{E}-02$ & $1.62 \mathrm{E}-02$ & 1.597 & 0.39212 & 0.21268 & 1.844 \\
\hline $\begin{array}{l}\text { Window (16 vs. NW) } \times \\
\text { Processing speed }\end{array}$ & -3.689 & 2.524 & -1.462 & -0.95223 & 0.9277 & -1.026 & $-3.18 \mathrm{E}-02$ & $1.62 \mathrm{E}-02$ & -1.956 & -0.19960 & 0.21324 & -0.936 \\
\hline \multicolumn{13}{|l|}{ Random effects } \\
\hline Item & 259.7 & 16.11 & NA & 14.03 & 3.745 & NA & 0.006276 & 0.07922 & NA & 1.521 & 1.233 & NA \\
\hline Subject & 1762.0 & 41.98 & NA & 413.78 & 20.342 & NA & 0.127835 & 0.35754 & NA & 15.435 & 3.929 & NA \\
\hline Residual & 1861.3 & 43.14 & NA & 252.13 & 15.879 & NA & 0.077082 & 0.27764 & NA & 13.292 & 3.646 & NA \\
\hline
\end{tabular}

${ }^{*} P<.05$. All $P$ values were obtained using the lmerTest package in $\mathrm{R}$

\section{Language ability}

As seen in Table 4, there were significant interactions between language ability and differences in WPM from the 4-character to the 8-character window, from the 12character to the 16-character window, and from the 16character to the no-window conditions. The interactions are depicted graphically in Fig. 1, which plots reading time measures for each window condition separately for the top half of the scores versus the bottom half of the scores on the language ability and processing speed composite measures. Readers with better language skills were more sensitive to the window size manipulation than were those with poorer language skills, demonstrating that readers with better language skills obtained greater processing benefit beyond the 12-character window relative to readers with poorer language skills. Similarly, there were significant interactions between language ability and differences in fixation duration from the 12-character window to the 16-character window and from the 16-character window to the no- window conditions. These results indicate that readers with better language skills make shorter fixations as window size increases, whereas readers with poorer language skills showed little modulation of fixation duration by window size. There were also interaction effects between language ability and differences in saccade amplitude from the 4-character to the 8-character window, from the 12-character to the 16-character window, and from the 16-character to the no-window size conditions. These interactions indicate that readers with better language skills showed larger saccades as window size increased, whereas readers with poorer language skills did not show much variation as window size increased. However, there was an interaction between language ability and differences in the number of regressive eye movements only from the 12-character to the 16character window. To sum up, the window size manipulation affected the eye movement patterns of readers with higher scores on measures of language ability more so than it did readers with lower scores on those measures. 
Fig. 1 Effects of individual difference measures on eye movement behaviors associated with window size. Left Graphs representing the relationship between language ability and eye movement behaviors, right graphs representing the relationship between oculomotor processing speed and eye movement behaviors. Error bars \pm 1 standard error
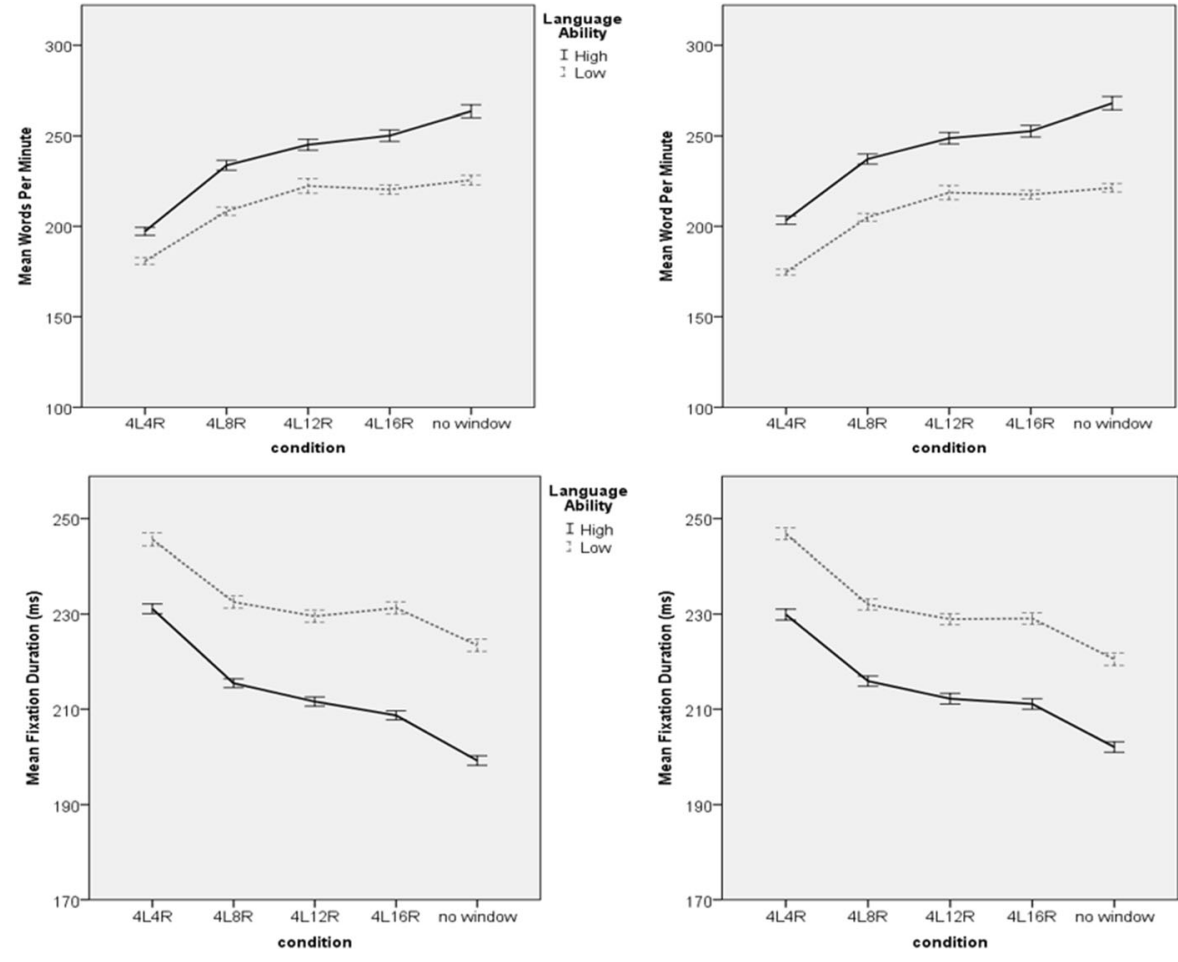

$\underset{\text { Processing }}{\text { Speed }}$

I Fast
S Slow
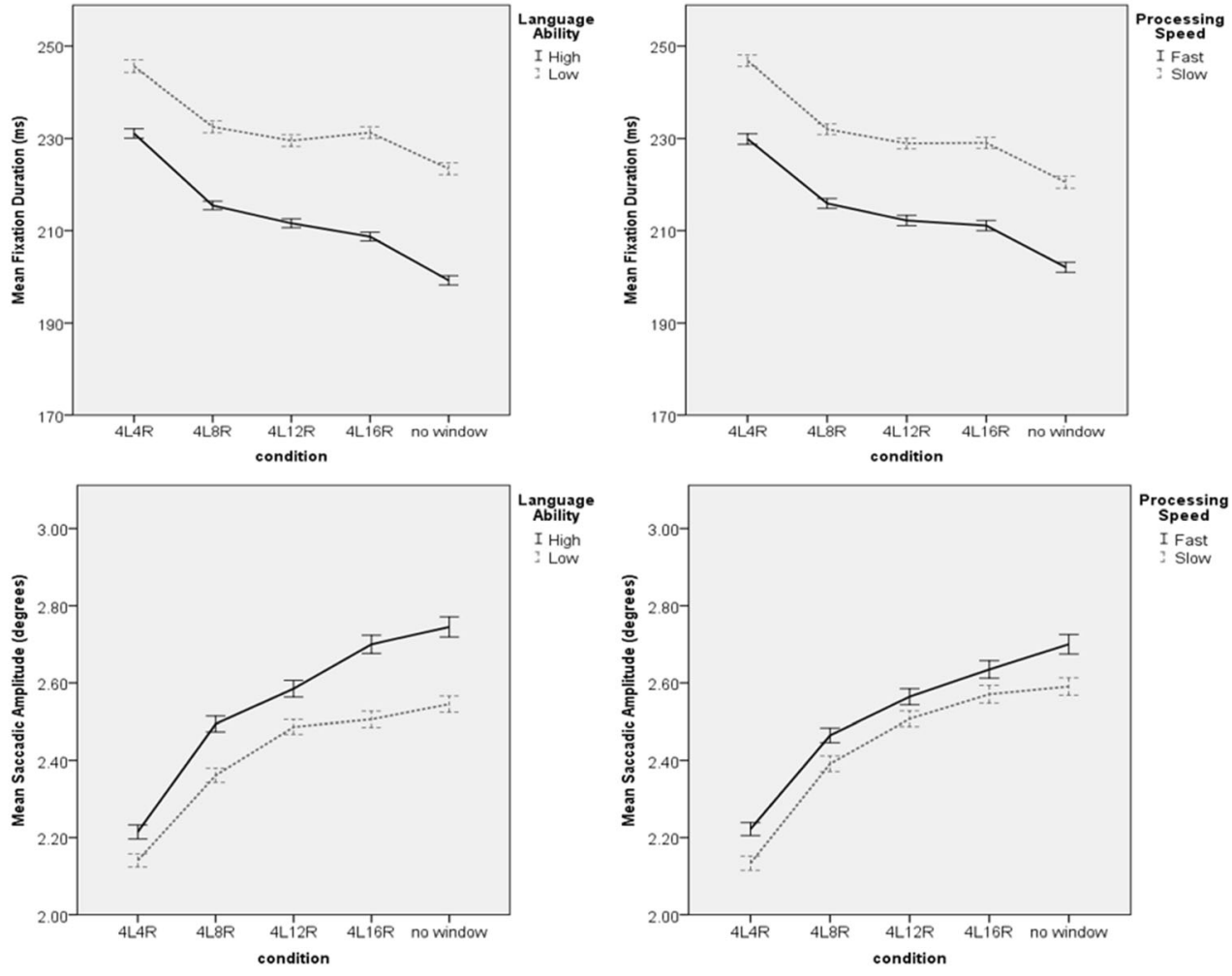

Processing
Speed I Fast
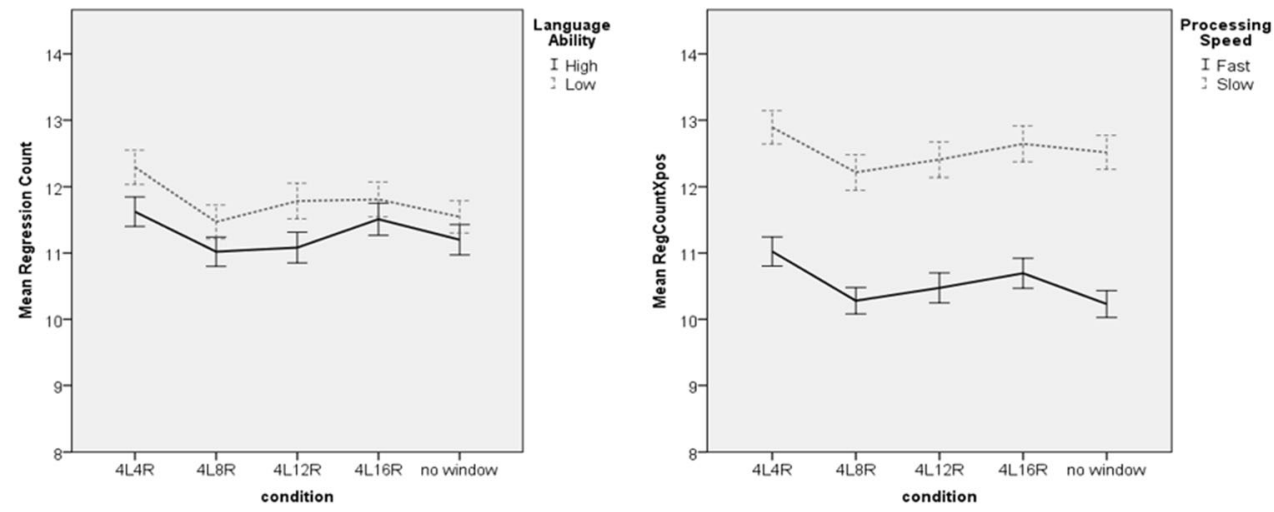

Oculomotor processing speed

There were no significant interactions between oculomotor processing speed and window size on WPM, fixation duration, saccade amplitude, and regressive eye movements. However, there was a marginally significant interaction between processing speed and saccade amplitude from the 16-character window to the 
no-window conditions, such that participants with faster processing speed had longer saccades as window size increased, whereas those with slower processing speed did not show any difference. In general, the effect of processing speed on eye movements was not strongly dependent on window size. Although participants with faster oculomotor processing speed tended to show faster reading rates, shorter fixation durations, and fewer regressive eye movements compared to those with slower processing speed, the effect of oculomotor processing speed was not strongly related to window size. ${ }^{1}$

\section{Discussion}

The current study was designed to investigate how individual differences in language ability and in oculomotor processing speed affect readers' perceptual span as assessed using the gaze-contingent moving window technique. Individual differences were measured using a large battery of cognitive tasks. Although we had hypothesized that individual differences in both language ability and oculomotor processing speed would be associated with variability in the size of readers' perceptual span, we found that language ability was a more reliable predictor of perceptual span than oculomotor processing speed. Specifically, we found that readers with better language ability had a larger perceptual span than did readers with poorer ability, consistent with previous studies using the moving window

\footnotetext{
${ }^{1}$ A reviewer suggested that both language ability and oculomotor processing speed might interact with window size, but that the effect of oculomotor processing speed might disappear when controlling for language ability. To test this idea, we generated simpler models that examined only the interaction between processing speed and window size, without the effect of language ability. There was evidence for an interaction in the analysis of reading rate [4-character vs 8 character $(\mathrm{t}=-2.497$, $P=.013)$, and 16-character vs no window $(\mathrm{t}=-2.366, P=$ $.018)$ ], suggesting that oculomotor processing speed might modulate perceptual span to some extent. However, there was no evidence of an interaction effect in analyses of fixation duration and saccade amplitude. Furthermore, a similar model that examined scores on the circle targeting task rather than the oculomotor speed of processing composite score showed no interaction effects at all. These results suggest that oculomotor processing speed is unlikely to be a critical factor that modulates the size of the perceptual span during reading.
}

technique (Häikiö et al., 2009; Rayner, 1986; Rayner, Murphy, Henderson, \& Pollatsek, 1989; Veldre \& Andrews, 2014). This result suggests that readers with superior language ability can effectively obtain additional processing benefit as the window size is increased. Highly skilled readers might be able to recognize the foveated word rapidly, leaving them with more attentional resources available to process information in rightward parafoveal regions (Henderson \& Ferreira, 1990; Veldre \& Andrews, 2014, 2015). Alternatively, highly skilled readers might benefit directly from the additional information available beyond the fovea. Particularly noteworthy, readers with high levels of language ability showed greater processing benefit in the no-window condition relative to the 16-character window condition, whereas readers with lower levels of language ability showed no additional improvement beyond the 12-character window. Veldre and Andrews (2014) also showed that readers' perceptual span can extend beyond 15 characters to the right of fixation. Whereas participants in Veldre and Andrews read single sentences, participants in the current study read paragraphs, suggesting that the modulation of readers' perceptual span by language ability is independent of text format.

Veldre and Andrews (2014) also found that highly skilled readers showed more processing disruption compared to less skilled readers when relatively small window sizes were used. They argued that the small window size prevented highly skilled readers from effectively using parafoveal information in planning saccades, which they are able to do under normal viewing conditions. However, the current study demonstrated that highly skilled readers showed faster reading rates and larger saccade amplitude relative to less skilled readers even in the smallest window condition. The discrepancy between these two results might be due to subtle differences in the window size manipulation. The smallest window size in the present study was four characters to the right of fixation, whereas the smallest window size in Veldre and Andrews was three characters to the right. The slightly larger window in the present study might have mitigated any processing disruptions among highly skilled readers. Note that the finding that greater processing disruption occurs in relatively small windows for highly skilled readers relative to less skilled readers has not been reported in other studies (e.g., Rayner, 1986). Another possible reason for this discrepancy might be that Veldre and Andrews used single sentences, whereas we used multi-sentence paragraphs. This difference in presentation may have changed the way readers process text in the smallest window size condition. Additional 
research should be focused on better understanding the nature of eye movements in relatively small windows.

Although individual differences in oculomotor processing speed influenced the properties of fixations and saccades during reading in general, this factor did not influence the size of the perceptual span as much as language ability did. The rapid naming task, which is believed to reflect oculomotor processing speed, has been identified as one of the strongest predictors of eye movements during reading (Kuperman \& Van Dyke, 2011). As shown in Table 2, the results in the present study also showed that RAN time (mean of the four RAN tasks) was correlated with eye movement measures such that participants with shorter RAN times had faster reading rates, shorter fixation durations, and fewer regressive eye movements. Similarly, participants with shorter times on the circle targeting task tended to have faster reading rates. Further, our composite score of oculomotor processing speed was a strong predictor of reading rates, fixation durations, and regression count (see Table 4), indicating that oculomotor speed of processing is related to eye movement behaviors during reading. The RAN and circle targeting tasks of course reflect cognitive processes in addition to oculomotor processing speed. As Kuperman \& Van Dyke (2011) pointed out, the RAN task also involves processes such as orthographic and/or phonological processing and serial oculomotor programming. Likewise, the circle targeting task used in the current study is also related to general processing speed as well as oculomotor processing speed. Given that natural reading requires rapid and sophisticated coordination of a variety of cognitive processes, it is reasonable to expect that eye movement behaviors during reading are correlated with general speed of processing as well as oculomotor programming.

Although individual differences in oculomotor speed of processing were correlated with eye movement measures during reading, this factor was not related to variability in reading rates across different window conditions. Our results showing that readers' perceptual spans are modulated by language ability but not processing speed suggests that oculomotor processing speed might not be a critical factor predicting variation in readers' perceptual span.

One particularly noteworthy finding of the current experiment is that scores on the ART showed substantial correlations with basic eye movement measures. Although previous studies have reported that ART scores are associated with language ability measured by a variety of offline tests (for a review, see Mol and Bus, 2011), the current experiment showed that readers with higher ART scores tend to have shorter fixation durations, longer saccades, and faster reading rates relative to those with lower ART scores. This finding suggests that the ART may be a good test to estimate not only readers' offline language ability but also their online eye movement patterns during reading. In addition, the $\mathrm{CRH}$ questionnaire showed strong correlations with several eye movement measures, such that readers with higher scores tended to have shorter fixation durations, longer saccades, and faster reading rates compared to readers with lower scores. This may be somewhat surprising, given that the CRH is a self-reported test and only includes five questions. Note that Acheson et al. (2008) did not find any correlations between measures of print exposure (ART and $\mathrm{CRH}$ ) and processing times using a self-paced reading paradigm. This difference between the current results and the findings reported by Acheson et al. (2008) may suggest that eye movement measures are more sensitive to variability in print exposure among readers, compared to self-paced reading. Although a great deal of work has shown that there are strong relationships between print exposure and language proficiency as assessed by offline tasks (see Mol and Bus, 2011, for a review), very little work has examined the relationship between print exposure and language proficiency using online measures such as eye tracking (cf. Moore \& Gordon, 2014). Our finding that print exposure is a reliable predictor of eye movement behaviors suggests that tasks like the ART and CRH can be useful in predicting individual variability in online reading.

In conclusion, the present study used a large battery of individual differences measures to obtain reliable estimates of readers' language ability and oculomotor processing speed. Although both of these constructs were related to readers' general eye movement behaviors, we found that individual differences in language ability were strongly associated with the size of readers' perceptual span, whereas there was a weaker relationship between oculomotor processing speed and perceptual span. These findings are consistent with the idea that readers with strong language skills are able to efficiently extract useful information from the parafovea during reading, which facilitates language processing.

Acknowledgments This research was supported by grants from the National Science Foundation (BCS-1151358). 


\section{Appendix 1}

Table 5 Correlation coefficients between individual difference measures. ART Author recognition test, $C R H$ comparative reading habits, $W I$ word identification, WA word attack, $L C$ listening comprehension, ORAN object rapid automatized naming, CRAN color rapid automatized naming, $N R A N$ number rapid automatized naming, $L R A N$ letter rapid automatized naming, $C T$ circle targeting

\begin{tabular}{|c|c|c|c|c|c|c|c|c|c|c|}
\hline & ART & $\mathrm{CRH}$ & WI & WA & $\mathrm{LC}$ & ORAN & CRAN & NRAN & LRAN & $\mathrm{CT}$ \\
\hline ART & 1 & $.51^{*}$ & $.53^{*}$ & .15 & $.45^{*}$ & -.15 & -.13 & -.05 & $-.27^{*}$ & .06 \\
\hline $\mathrm{CRH}$ & & 1 & $.30^{*}$ & .12 & $.36^{*}$ & -.20 & -.01 & .01 & -.16 & -.07 \\
\hline WI & & & 1 & $.56^{*}$ & $.51^{*}$ & $-.26^{*}$ & $-.32 *$ & $-.31^{*}$ & $-.42 *$ & .11 \\
\hline WA & & & & 1 & $.34 *$ & $-.33^{*}$ & $-.29^{*}$ & $-.31^{*}$ & $-.36^{*}$ & -.03 \\
\hline $\mathrm{LC}$ & & & & & 1 & -.18 & -.06 & -.14 & -.18 & .07 \\
\hline ORAN & & & & & & 1 & $.71^{*}$ & $.50^{*}$ & $.53^{*}$ & $.24 *$ \\
\hline CRAN & & & & & & & 1 & $.59^{*}$ & $.66^{*}$ & $.25^{*}$ \\
\hline NRAN & & & & & & & & 1 & $.87 *$ & .18 \\
\hline LRAN & & & & & & & & & 1 & .15 \\
\hline CT & & & & & & & & & & 1 \\
\hline
\end{tabular}

$* P<.05$

\section{Appendix 2}

Table 6 Component loadings for the principal components analysis of the individual difference measures

\begin{tabular}{lll}
\hline & \multicolumn{2}{l}{ Component } \\
\cline { 2 - 3 } ID measures & $\begin{array}{l}\text { 1 (oculomotor } \\
\text { processing speed) }\end{array}$ & 2 (language ability) \\
\hline Author recognition test & .032 & .779 \\
Comparative reading habits & .024 & .667 \\
Word identification & -.202 & .790 \\
Word attack & -.305 & .536 \\
Listening comprehension & -.025 & .728 \\
Object RAN & .688 & -.295 \\
Color RAN & .851 & -.085 \\
Number RAN & .848 & -.045 \\
Letter RAN & .848 & -.237 \\
Circle targeting task & .534 & 228 \\
Explained variance & $37 \%$ & $23 \%$ \\
\hline
\end{tabular}

\section{References}

Acheson, D. J., Wells, J. B., \& MacDonald, M. C. (2008). New and updated tests of print exposure and reading abilities in college students. Behavior Research Methods, 40, 278-289.

Apel, J. K., Henderson, J. M., \& Ferreira, F. (2012). Targeting regressions: Do readers pay attention to the left? Psychonomic Bulletin \& Review, 19, 1108-1113.

Ashby, J., Rayner, K., \& Clifton, C., Jr. (2005). Eye movements of highly skilled and average readers: Differential effects of frequency and predictability. The Quarterly Journal of Experimental Psychology Section A, 58A, 1065-1086.

Ashby, J., Yang, J., Evans, K. H. C., \& Rayner, K. (2012). Eye movements and the perceptual span in silent and oral reading. Attention, Perception and Psychophysics, 74, 634-640.

Bates, D., Maechler, M., \& Bolker, B (2012). Ime4: Linear mixed-effects models using $S 4$ classes (R package version 0.999999-0). Retrieved from http://CRAN.R-project.org/

Cunningham, A. E., \& Stanovich, K. E. (1990). Assessing print exposure and orthographic processing skill in children: A quick measure of reading experience. Journal of Educational Psychology, 82, 733-740.

Cunningham, A. E., \& Stanovich, K. E. (1991). Tracking the unique effects of print exposure in children: Associations with vocabulary, general knowledge, and spelling. Journal of Educational Psychology, 83, 264-274.

Cunningham, A. E., \& Stanovich, K. E. (1997). Early reading acquisition and its relation to reading experience and ability 10 years later. Developmental Psychology, 33, 934-945. 
Cutting, L. E., \& Denckla, M. B. (2001). The relationship of rapid serial naming and word reading in normally developing readers: An exploratory model. Reading and Writing, 14, 673-705.

Denckla, M. B., \& Rudel, R. G. (1974). Rapid "automatized” naming of pictured objects, colors, letters, and numbers by normal children. Cortex, 10, 186-202.

Denckla, M. B., \& Rudel, R. G. (1976). Rapid 'automatized' naming (RAN): Dyslexia differentiated from other learning disabilities. Neuropsychologia, 14, 471-479.

Eden, G. F., Stein, J. F., Wood, H. M., \& Wood, F. B. (1994). Differences in eye movements and reading problems in dyslexic and normal children. Vision Research, 34, 1345-1358.

Eskanazi, B., \& Diamond, S. P. (1983). Visual exploration of nonverbal material by dyslexic children. Cortex, 19, 345-370.

Griffin, D. C., Walton, H. N., \& Ives, V. (1974). Saccades as related to reading disorders. Journal of Learning Disabilities, 7, 310-316.

Häikiö, T., Bertram, R., Hyönä, J., \& Niemi, P. (2009). Development of the letter identity span in reading: Evidence from the eye movement moving window paradigm. Journal of Experimental Child Psychology, 102, 167-181.

Henderson, J. M., \& Ferreira, F. (1990). The effects of foveal difficulty on the perceptual span in reading: Implications for attention and eye movement control. Journal of Experimental Psychology: Learning, Memory, and Cognition, 16, 417-429.

Jared, D., Levy, B. A., \& Rayner, K. (1999). The role of phonology in the activation of word meanings during reading: Evidence from proofreading and eye movements. Journal of Experimental Psychology: General, 128, 219-264.

Juola, J. F., Haugh, D., Trast, S., Ferraro, F. R., \& Liebhaber, M. (1987). Reading with and without eye movements. In J. K. O'Regan \& A. Levy-Schoen (Eds.), Eye movements: From Physiology to Cognition (pp. 499-508). North-Holland: Elsevier.

Kail, R., \& Hall, L. K. (1994). Processing speed, naming speed and reading. Developmental Psychology, 30, 949-954.

Kail, R., Hall, L. K., \& Caskey, B. L. (1999). Processing speed, exposure to print, and naming speed. Applied Psycholinguistics, 20, 303-314.

Kulp, M. T., \& Schmidt, P. P. (1996). Effect of oculomotor and other visual skills on reading performance: A literature review. Optometry and Vision Science, 73, 283-292.

Kuperman, V., \& Van Dyke, J. A. (2011). Effects of individual differences in verbal skills on eye-movement patterns during sentence reading. Journal of Memory and Language, 65, 42-73.

Kuznetsova, A., Brockhoff, P. B., \& Christensen, R. H. B. (2013). ImerTest: Tests for random and fixed effects for linear mixed effect models (Imer objects of lme4 package).

Lefton, L. A., Nagle, R. J., Johnson, G., \& Fisher, D. F. (1979). Eye movement dynamics of good and poor readers: Then and now. Journal of Reading Behavior, 11, 319-327.

McCardle, P., \& Pugh, K. (2009). How children learn to read: current issues and new directions. In The integration of cognition, neurobiology and genetics of reading and dyslexia research and practice. Oxford: Psychology.

McConkie, G. W., \& Rayner, K. (1975). The span of the effective stimulus during a fixation in reading. Perception \& Psychophysics, 17, $578-586$.

Mol, S. E., \& Bus, A. G. (2011). To read or not to read: A meta-analysis of print exposure from infancy to early adulthood. Psychological Bulletin, 137, 267-296.

Moore, M., \& Gordon, P. C. (2014). Reading ability and print exposure: Item response theory analysis of the Author Recognition Test. Behavioral Research Methods. doi:10.3758/s13428-014-0534-3

Olson, R. K., Kliegl, R., \& Davidson, B. J. (1983). Dyslexic and normal readers' eye movements. Journal of Experimental Psychology: Human Perception and Performance, 9, 816-825.
Palmer, J., MacLeod, C. M., Hunt, E., \& Davidson, J. E. (1985). Information processing correlates of reading. Journal of Memory and Language, 24, 59-88.

Pavlidis, G. T. (1981). Do eye movements hold the key to dyslexia? Neuropsychologia, 19, 57-64.

Pavlidis, G. T. (1983). The "dyslexia syndrome" and its objective diagnosis by erratic eye movements. In K. Rayner (Ed.), Eye movements in reading: Perceptual and language processes (pp. 441-466). New York: Academic

Powell, D., Stainthorp, R., Stuart, M., Garwood, H., \& Qunlan, P. (2007). An experimental comparison between rival theories of rapid automatized naming performance and its relationship to reading. Journal of Experimental Child Psychology, 98, 46-68.

R Development Core Team. (2011). R: A language and environment for statistical computing. Vienna, Austria: R Foundation for Statistical Computing. Retrieved from http://www.r-project.org/

Rayner, K. (1975). The perceptual span and peripheral cues in reading. Cognitive Psychology, 7, 65-81.

Rayner, K. (1985). The role of eye movements in learning to read and reading disability. Remedial and Special Education, 6, 53-60.

Rayner, K. (1986). Eye movements and the perceptual span in beginning and skilled readers. Journal of Experimental Child Psychology, 41, 211-236.

Rayner, K. (2009). Eye movements and attention in reading, scene perception, and visual search. Quarterly Journal of Experimental Psychology, 62, 1457-1506.

Rayner, K. (2014). The gaze-contingent moving window in reading: Development and review. Visual Cognition, 22, 242-258.

Rayner, K., Murphy, L. A., Henderson, J. M., \& Pollatsek, A. (1989). Selective attentional dyslexia. Cognitive Neuropsychology, 6, 357378.

Rayner, K., Slattery, T. J., \& Bélanger, N. N. (2010). Eye movements, the perceptual span, and reading speed. Psychonomic Bulletin \& Review, 17, 834-839.

Reichle, E. D., Pollatsek, A., Fisher, D. L., \& Rayner, K. (1998). Toward a model of eye movement control in reading. Psychological Review, $105,125-157$.

Rieben, L., \& Perfetti, C. A. (Eds.). (1991). Learning to read: Basic research and its implications. Hillsdale, $\mathrm{NJ}$ : Erlbaum.

Savage, R., Pillay, V., \& Melidona, S. (2007). Deconstructing rapid naming: Component processing and the prediction of reading difficulties. Learning and Individual Differences, 17, 129-146.

Stanley, G., Smith, G. A., \& Howell, E. A. (1983). Eye movements and sequential tracking in dyslexic and control children. British Journal of Psychology, 74, 181-187.

Stanovich, K. E., \& West, R. F. (1989). Exposure to print and orthographic processing. Reading Research Quarterly, 24, 402433.

Stanovich, K. E., West, R. F., \& Harrison, M. R. (1995). Knowledge growth and maintenance across the life span: The role of print exposure. Developmental Psychology, 31, $811-826$.

Swanson, H. L., Trainin, G., Necoechea, D. M., \& Hammill, D. D. (2003). Rapid naming, phonological awareness, and reading: A meta-analysis of the correlation evidence. Review of Educational Research, 73, 407-440.

Veldre, A., \& Andrews, S. (2014). Lexical quality and eye movements: Individual differences in the perceptual span of skilled adult readers. The Quarterly Journal of Experimental Psychology, 67, 703-727.

Veldre, A., \& Andrews, S. (2015). Parafoveal lexical activation depends on skilled reading proficiency. Journal of Experimental Psychology: Learning, Memory, and Cognition, 41, 586-589. 
Venables, W. N., \& Ripley, B. D. (2002). Modern applied statistics with S (4th ed.). New York: Springer.

West, R. F., Stanovich, K. E., \& Mitchell, H. R. (1993). Reading in the real world and its correlates. Reading Research Quarterly, $28,35-50$.
Wolf, M., \& Bowers, P. G. (1999). The double-deficit hypothesis for the developmental dyslexias. Journal of Educational Psychology, 91, 415-438.

Woodcock, R. W. (2011). Wookcock reading mastery tests third edition manual. Oxford: Pearson. 